\title{
Urinary kidney injury molecule-1 as an early diagnostic biomarker of obstructive acute kidney injury and development of a rapid detection method
}

\author{
YINGLI JIN ${ }^{1}$, XIAONA SHAO ${ }^{2}$, BO SUN $^{2}$, CHUNSHENG MIAO $^{2},{\text { ZHENGQIANG } \text { LI }^{3} \text { and YAN SHI }}^{2}$ \\ ${ }^{1}$ Department of Pharmacology, College of Basic Medical Science, Jilin University; \\ ${ }^{2}$ Department of Experimental Pharmacology and Toxicology, School of Pharmacy, Jilin University, \\ Changchun, Jilin 130021; ${ }^{3}$ Key Laboratory for Molecular Enzymology and Engineering, \\ Ministry of Education, Jilin University, Changchun, Jilin 130012, P.R. China
}

Received October 23, 2015; Accepted November 17, 2016

DOI: $10.3892 / \mathrm{mmr} .2017 .6103$

\begin{abstract}
The aim of the present study was to investigate whether urinary kidney injury molecule-1 (KIM-1) presents a suitable early diagnostic biomarker of obstructive nephropathy-induced acute kidney injury (AKI), and to develop a rapid detection method for urinary KIM-1. Obstructive AKI was induced in an experimental rat model by a unilateral ureteral obstruction (UUO) operation. Macro- and micromorphological kidney alterations were determined by visual observation and hematoxylin and eosin (HE) staining, respectively. Kidney functions were evaluated by detecting urea nitrogen and creatinine levels in rat urine and blood. Urinary KIM-1 levels were measured using an enzyme-linked immunosorbent assay, and the protein expression levels of KIM-1, $\alpha$-smooth muscle actin $(\alpha$-SMA) and vimentin in kidney tissues were detected using immunohistochemical assays. In order to measure KIM-1 levels, colloidal gold immunochromatographic strips were developed based on the colloidal gold immunochromatographic assay. The results indicated that KIM-1 levels were significantly higher in the UUO group when compared with the Sham group. KIM-1 levels in the urine and kidney tissues exhibited a time-dependent increase, together with increasing obstructive AKI in the UUO group. In addition, KIM-1 levels were demonstrated to be a more sensitive biomarker of early obstructive AKI, when compared with $\alpha$-SMA and vimentin. A colloidal gold-based immunochromatographic strip was developed, whereby the detection
\end{abstract}

Correspondence to: Dr Yan Shi, Department of Experimental Pharmacology and Toxicology, School of Pharmacy, Jilin University, 1266 Fujin Road, Changchun, Jilin 130021, P.R. China

E-mail: shiyan0431@126.com

Key words: obstructive acute kidney injury, kidney injury molecule-1, early diagnostic biomarker, rapid diagnosis, colloidal gold immunochromatographic strip of urinary KIM-1 could be completed within 5-10 min. In conclusion, results of the present study demonstrated that urinary KIM-1 may be a valuable biomarker for the early diagnosis of obstructive AKI, and the use of a colloidal gold immunochromatographic strip may be a promising method for the rapid detection of urinary KIM-1.

\section{Introduction}

Acute kidney injury (AKI), previously defined as acute renal failure, is a common and devastating complication among hospitalized patients with acute illnesses, particularly among those with a life-threatening illness (1). AKI is characterized by aberrant alterations in kidney function within hours to days following onset (2). According to a systematic study reviewing AKI incidence between the years of 2004 and 2012, the worldwide incidence rates in adults and children were 21.6 and $33.7 \%$, respectively, and the corresponding mortality rates were 23.9 and $13.8 \%$, respectively (3). Although clinicians have made significant progress in AKI treatment (4), the prognosis of AKI remains poor. This may be due, in part, to the lack of effective strategies that enable early diagnosis of AKI, which may lead to neglecting appropriate opportunities for intervention during the early stages of the disease (5). Therefore, the development of efficient biomarkers for the early diagnosis of AKI has become an important area of research worldwide during the last decade (6).

Kidney injury molecule-1 (KIM-1) is a type I transmembrane glycoprotein primarily expressed on the surface of $\mathrm{T}$ cells, and possesses two extracellular domains (7). KIM-1 expression is low in normal kidneys, but is significantly increased in proximal tubule cells following kidney injury. Upon injury, the extracellular domains of KIM-1 separate from the cell surface and enter the urine through a metalloproteinase-dependent process (7). It has been demonstrated that urinary KIM-1 concentration is markedly increased within $h$ following kidney injury (8). Therefore, detecting the level of urinary KIM-1 may potentially be an effective method for the early diagnosis of AKI. Urinary KIM-1 levels have, thus far, been permitted by the US Food and Drug Administration to be 
used as an early diagnostic biomarker of drug-induced AKI in preclinical studies (5).

However, whether urinary KIM-1 may be used as an early diagnostic biomarker of obstructive AKI requires further validation. Obstructive AKI is the nephropathy-induced form of AKI caused by congenital or acquired urinary tract obstruction (9). In the present study, the potential of urinary KIM-1 as an early diagnostic biomarker of obstructive AKI was investigated by establishing a unilateral ureteral obstruction (UUO) rat model. In addition, a colloidal gold-based immunochromatographic strip for the detection of KIM-1 was developed, with the aim of providing a novel technique for the rapid diagnosis of $\mathrm{AKI}$.

\section{Materials and methods}

Reagents. Chloral hydrate was bought from Qilu Pharmaceutical Co. Ltd. (Jinan, China). A hematoxylin and eosin (HE) staining kit was obtained from Beijing Disinbio Science Technology Co. Ltd. (Beijing, China). A KIM-1 enzyme-linked immunosorbent assay (ELISA) kit was purchased from R\&D Systems, Inc. (Minneapolis, MN, USA). Immunohistochemistry (IHC) kits for KIM-1, $\alpha$-SMA and vimentin were obtained from BD Biosciences (Franklin Lakes, NJ, USA). Human KIM-1 monoclonal antibody (McAb; cat. no., BAF1750), goat anti-mouse IgG (cat. no., BAF007) and the goat anti-human polyclonal antibody (PcAb) KIM-1 (cat. no., AF1750) were obtained from R\&D Systems, Inc. A fiberglass membrane SB06, polyester fiber membrane VL78, absorbent paper $\mathrm{CH} 27$ and a PVC plastic back plate were purchased from Shanghai Goldbio Technology Co., Ltd. (Shanghai, China), and the nitrocellulose membrane was obtained from Merck Millipore (Darmstadt, Germany).

Animals and design. Wistar rats (20 male and 20 female; age, 6-8 weeks; weight, 180-220 g) were provided by the Experimental Animal Center of Jilin University (Changchun, China). All rats were housed in a pathogen-free facility with free access to water and standard laboratory chow. The temperature was maintained at $20-25^{\circ} \mathrm{C}$ and a $12 / 12 \mathrm{~h}$ light/dark cycle was simulated. Prior to establishment of the UUO model, the rats were randomly assigned into four equal groups, consisting of a Sham group and three UUO groups. The Sham group was used as the control group, whereby ligation and severing of the ureter was not performed during the modeling processes. The three UUO groups were UUO1, UUO3 and UUO7, and consisted of rats that were sacrificed at 1,3 and 7 days post-UUO, respectively. The mental state and diet of rats in each group was recorded every day, and body weight was measured before and after UUO modeling. Following UUO modeling, the body weights were determined before the rats were sacrificed. The present study was approved by the Animal Ethical Committee of Jilin University (Changchun, China). Following blood collection, rats were sacrificed by cervical dislocation. Rats in UUO1, UUO3 and UUO7 groups were sacrificed at 1,3 and 7 days post-UUO, respectively. Those in the Sham group were sacrificed at 7 days after the modeling operation.

UUO modeling. Rats were first acclimated to laboratory conditions for 7 days and fasted for $12 \mathrm{~h}$, before they were anesthetized by intraperitoneal injection of $10 \%$ chloral hydrate at a dose of $2 \mathrm{mg} / \mathrm{kg}$ and fixed in a supine position. On the left-hand side of the middle abdomen, a 2-cm lengthwise incision was made. The left ureter was isolated and ligated in the renal pelvis and in the upper third of the ureter before it was severed between the two ligatures. The incised abdominal skin was subsequently sutured layer-by-layer. This procedure was conducted under sterile conditions. For rats in the sham group, the same procedure was performed without ligating or severing the ureter.

Collection of urine and blood, and evaluation of kidney function. One day prior to sacrifice, rats were placed in metabolic cages and their urine was collected over a period of $24 \mathrm{~h}$. During this process, rats were fasted but had free access to water. The total volume of urine from each rat over $24 \mathrm{~h}$ was measured, and $3 \mathrm{ml}$ of the urine was centrifuged at $400 \mathrm{x} g$ for $10 \mathrm{~min}$ at room temperature. The supernatant was collected and transferred to Eppendorf tubes, before storing at $-20^{\circ} \mathrm{C}$ for downstream analysis. A total of $5 \mathrm{ml}$ blood was harvested from vessels near the eyeball by removing the eyeball, before centrifuging at $600 \mathrm{~g}$ for $10 \mathrm{~min}$ at room temperature. Serum was collected and stored at $-20^{\circ} \mathrm{C}$ until further analysis. Kidney function was evaluated by examining the level of urea nitrogen and creatinine in rat urine and blood, which was performed using a 7150 Biochemical Analyzer (Hitachi, Tokyo, Japan) according to the manufacturer's instructions.

Kidney tissue specimen collection. Both kidneys from each rat were obtained immediately following sacrifice and were analyzed for morphological alterations in appearance and internal structures. Following removal of the capsule, kidneys were rinsed with saline and weighed using scales. They were then divided into several pieces, quickly frozen with liquid nitrogen and stored at $-80^{\circ} \mathrm{C}$. Prior to analysis, kidney tissues were paraffin-embedded and divided into sections $(3 \mu \mathrm{m}$ in thickness) according to the description by Wu et al (10).

Histological examination of kidney damage. Following deparaffinization and rehydration, tissue sections were stained with $\mathrm{HE}$ for histopathological observation using a Hematoxylin-Eosin Staining kit. Histological alterations in the renal tubule interstitium were assessed according to the quantitative measurements of tubular damage, characterized by tubular epithelial swelling, degeneration, necrosis, tubular ectasia, inflammatory cell infiltration and tubular interstitial fibrosis (11). Ten kidney sections from each rat were randomly selected and evaluated at x200 magnification. The degree of kidney damage was scored according to the following criteria described by Leelahavanichkul et al (11): 0, normal; 1, area of damage $=<25 \%$ of tubules; 2 , area of damage $=25-50 \%$ of tubules; 3 , area of damage $=50-75 \%$ of tubules; and 4 , area of damage $=75-100 \%$ of tubules.

ELISA. Urinary KIM-1 levels were detected using the KIM-1 ELISA kit according to the manufacturer's instructions (R\&D Systems, Inc.). Urine samples with coating buffer were added to 96 -well plates $\left(100 \mu \mathrm{l} /\right.$ well) and incubated overnight at $4^{\circ} \mathrm{C}$. The remaining protein-binding sites were then blocked by incubating samples for $1.5 \mathrm{~h}$ with blocking buffer containing 
$2 \%$ fetal bovine serum at $37^{\circ} \mathrm{C}$. Primary mouse anti-rat KIM-1 monoclonal antibodies (dilution, 1:1,000) and goat anti-mouse IgG secondary antibodies (dilution, 1:2,000) were added in order to bind specifically with the target antigen. Following treatment with 3,3-diaminobenzidene (DAB) solution from the ELISA kit and stop buffer, the plates were read at $490 \mathrm{~nm}$ using a microplate reader (DG5031, Shanghai Jinggong Industrial Co., Ltd, Shanghai, China).

IHC analysis. Protein expression of KIM-1, $\alpha-$ SMA and vimentin in kidney tissues was detected using an IHC kit according to the manufacturer's instructions (BD Biosciences). Following deparaffinization and rehydration, tissue sections were incubated with $1 \% \mathrm{H}_{2} \mathrm{O}_{2}$ for 5-10 min to inactivate endogenous peroxidases, and the antigen was retrieved by microwaving for $5 \mathrm{~min}$ in citrate buffer. The sections were subsequently blocked with goat serum for $20 \mathrm{~min}$ at room temperature and incubated with primary antibodies (mouse anti-rat KIM-1 McAb, dilution, 1:200, BAF1750, R\&D Systems, Inc.; mouse anti-rat $\alpha$-SMA McAb, dilution, 1:200, BSM-33187M, BIOSS, Beijing, China; mouse anti-rat McAb, dilution, 1:200, V5255, Sigma-Aldrich; Merck-Millipore, Darmstadt, Germany) overnight at $4^{\circ} \mathrm{C}$. Sections were then incubated with the ready-to-use secondary antibody conjugated to horseradish peroxidase (SP-0022, BIOSS) at room temperature for $40 \mathrm{~min}$. After rinsing with phosphate-buffered saline, the sections were incubated in streptaridin-peroxidase mixture (BIOSS) at room temperature for $20 \mathrm{~min}$, followed by DAB solution for $10 \mathrm{~min}$ at room temperature. The sections were then counterstained with hematoxylin and mounted with neutral balsam. Brown staining observed under the microscope indicated KIM-1, $\alpha$-SMA or vimentin protein expression. Semi-quantitative analysis was performed according to staining intensity and area, and was scored as 0 (no staining or extremely weak), 1 (mild, staining area $<25 \%$ ), 2 (moderate, staining area involving 26-50\%), 3 (strong, staining area involving $51-75 \%$ ) or 4 (strong, staining area $>76 \%$ ).

Preparation of colloidal gold-McAb KIM-1 conjugates. Colloidal gold with a diameter of $40 \mathrm{~nm}$ was purchased from Shanghai Kinbio Tech Co., Ltd (Shanghai, China). The optimal $\mathrm{pH}$ for binding colloidal gold with protein was equal to the isoelectric point $(\mathrm{pI})$ of the protein plus 0.5 (12). The pI of human KIM-1 McAb was 7.4, so the optimal $\mathrm{pH}$ for binding colloidal gold with human KIM-1 McAb was 7.9. The minimal McAb KIM-1/colloidal gold ratio at which colloidal gold is stabilized with McAb KIM-1 was determined by mixing $100 \mu \mathrm{l}$ colloidal gold ( $\mathrm{pH}$ 7.9) with different volumes (0-50 $\mu \mathrm{l} ; 5 \mu \mathrm{l}$ increments) of McAb KIM-1 (100 $\mu \mathrm{g} / \mathrm{ml})$. At the minimal ratio or higher, the mixtures will maintain their red color due to the stabilized colloidal gold by using enough McAb KIM-1, or the color will change from red to blue accompanied with the deposition of redundant colloidal gold. Therefore, the minimal McAb KIM-1/colloidal gold ratio can be determined by observing the color change of these mixtures. The actual volume of McAb KIM-1 used was the minimum calculated volume, based on the minimal ratio plus $10 \%$ of this volume. Colloidal gold and KIM-1 $\mathrm{McAb}$ were mixed according to the actual usage ratio to obtain the colloidal gold-McAb KIM-1 conjugate solution.
This was then purified through a two-step centrifugation. The conjugate solution was centrifuged at $600 \mathrm{x} g$ for $10 \mathrm{~min}$ at $4^{\circ} \mathrm{C}$, and the supernatant was collected and centrifuged again at $8,000 \times \mathrm{g}$ for $60 \mathrm{~min}$ at $4^{\circ} \mathrm{C}$. The precipitate was harvested and resuspended with $0.01 \mathrm{M}$ phosphate-buffered saline supplemented with $0.02 \% \mathrm{NaN}_{3}$ and $1 \%$ bovine serum albumin, whereby the purified colloidal gold-McAb KIM-1 conjugate solution was obtained.

Development of a colloidal gold-based immunochromatographic strip. The immunochromatographic strip consisted of five sections, which were sourced from Shanghai Goldbio Technology Co., Ltd., unless stated otherwise. The section consisted of the following components: A sample pad fiberglass membrane (SB06), a conjugate pad polyester fiber membrane (VL78), an analyzing nitrocellulose membrane (Merck Millipore), an absorbent pad (CH27) and a plastic back plate. The conjugate pad was pretreated with the purified colloidal gold-McAb KIM-1 conjugates, and then dried in an incubator at $37^{\circ} \mathrm{C}$ for $30 \mathrm{~min}$. A control (C) line and test (T) line were marked on the analyzing membrane with goat anti-mouse IgG (2 mg/ml; BAF007, R\&D Systems, Inc.) and goat anti-human PcAb KIM-1 (200 $\mu \mathrm{g} / \mathrm{ml}$, dilution, 1:200, AF1750, R\&D Systems, Inc.), respectively. The sample pad, pretreated conjugate pad, analyzing membrane and absorbent pad were pasted on a plastic back plate. The detection limit of the colloidal gold-based immunochromatographic strip was determined by detecting normal urine specimens supplemented with KIM-1 at the final concentrations of 0,50 and $100 \mathrm{ng} / \mathrm{ml}$, and 1 and $10 \mu \mathrm{g} / \mathrm{ml}$, respectively.

Statistical analysis. Statistical analyses of rat weight, kidney weight, kidney function and ELISA assays among the experimental groups were performed using one-way analysis of variance. Comparisons between two groups were further analyzed with the Student-Newman-Keuls post-hoc test. All the statistical analyses were performed with the SPSS software program (version, 19.0; IBM SPSS, Armonk, NY, USA). $\mathrm{P}<0.05$ was considered to indicate a statistically significant difference.

\section{Results}

Obstructive AKI was successfully induced by the UUO operation. Rats in all experimental groups survived the UUO operation, however UUO rats exhibited signs of depression, which was accompanied by a distinct reduction in food intake. The average body weights of rats in the UUO3 and UUO7 groups were significantly decreased following UUO modeling $(\mathrm{P}=0.036$ and $\mathrm{P}=0.048$, respectively), and the decrease was time-dependent (Fig. 1A). Conversely, the weight of the left kidney in UUO groups was consistently higher than that of the right kidney (Fig. 1B), which reached statistical significance in the UUO7 group $(\mathrm{P}=0.032)$. By contrast, the body and kidney weight of the Sham group demonstrated no significant alterations before and after UUO modeling ( $\mathrm{P}>0.05)$.

With regards to kidney morphology, the left kidney in rats from all UUO groups appeared larger and was darker in color, when compared with corresponding right kidney, and the left ureter above the ligatures exhibited obvious enlargements. 


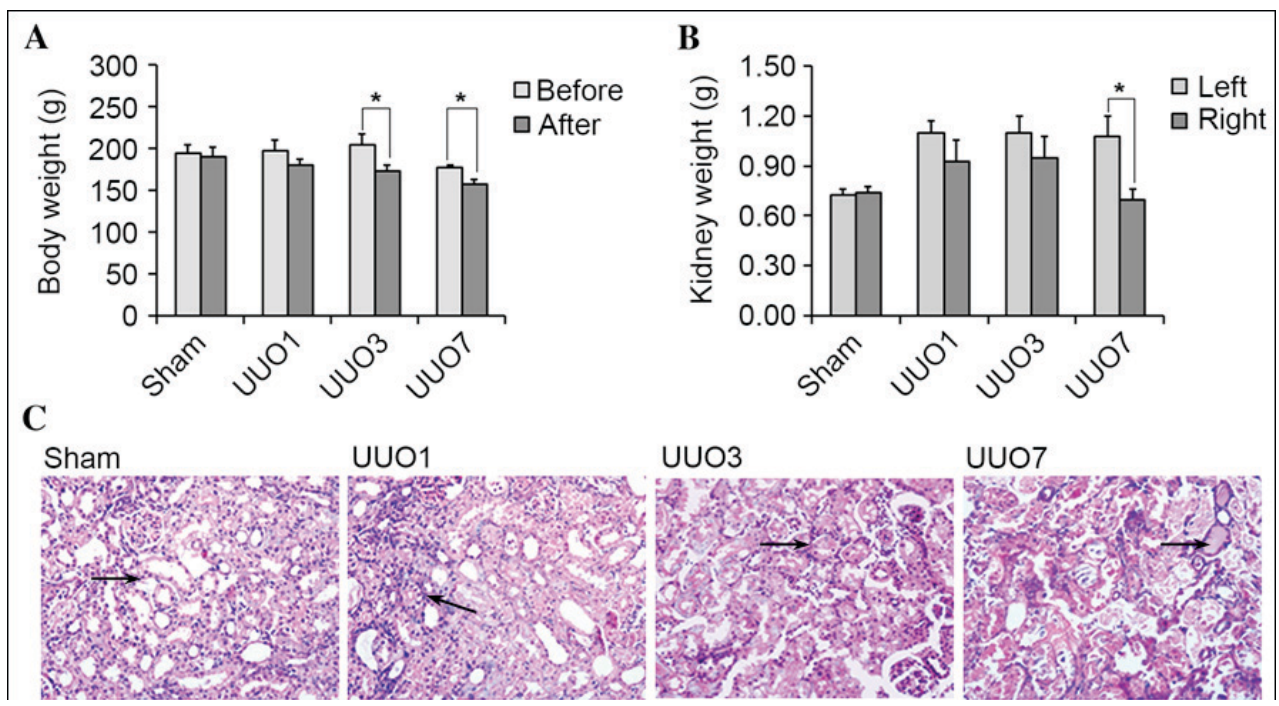

Figure 1. Alterations in body weight, kidney weight and micro-morphology following UUO operation. (A) The body weight of rats at 1 (UUO1), 3 (UUO3) and 7 (UUO7) days following UUO decreased following UUO operation ( ${ }^{*} \mathrm{P}<0.05$ vs. before UUO). (B) The weight of the left kidney was greater than that of the right kidney in the UUO groups following the UUO operation (" $\mathrm{P}<0.05$ vs. the left kidney). (C) Kidney injury at the micro-morphological level increased in a time-dependent manner in the UUO group as shown by the arrows. In the Sham group, no clear damage was observed; In UUO1 group, kidney tubules were enlarged marginally and the renal interstitium was infiltrated by few inflammatory cells. In the UUO3 group, alterations such as renal interstitial edema, inflammatory cell infiltration and vacuolar degeneration of tubular epithelial cells were clear. In the UUO7 group, the damage was progressed further, which was characterized by tubular epithelial necrosis, renal interstitial broadening, and tubular interstitial fibrosis. UUO, unilateral ureteral obstruction.

A

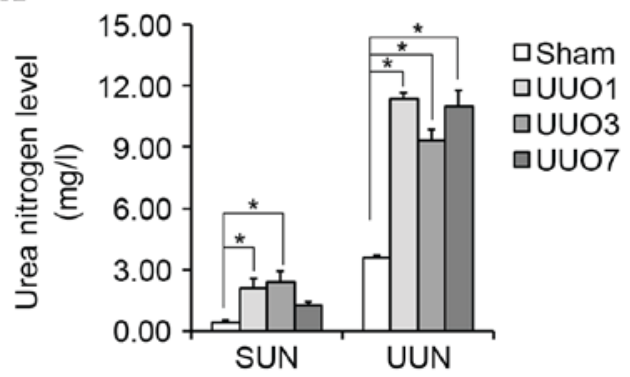

B

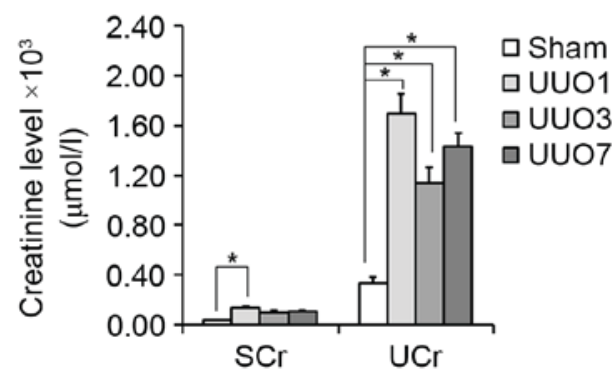

Figure 2. Kidney dysfunction was induced by UUO operation. (A) Urea nitrogen levels in the serum (SUN) and urine (UUN) were higher in rats at 1 (UUO1), 3 (UUO3) and 7 (UUO7) days following UUO when compared with the Sham group. (B) Serum (SCr) and urine creatinine (UCr) levels were higher in the UUO group when compared with the Sham group. " $\mathrm{P}<0.05$ vs. Sham group. UUO, unilateral ureteral obstruction.

Following a transverse cut in the kidney, ischemic injury was observed in the left kidney, particularly in the renal cortex. Renal pelvis and calyces were enlarged and were filled with turbid brown urine. Renal papillae were smooth and disappeared. The renal parenchyma was thinner with an indistinct boundary between the renal cortex and the medulla. The above alterations in the appearance and internal structures of left kidneys in UUO groups were time-dependent. By contrast, rat kidneys in the Sham group exhibited no substantial alterations (data not shown).

Histopathological observations suggested that kidneys in the Sham group demonstrated no obvious damage and were scored as 0 (data not shown). In UUO groups, kidneys exhibited mild damage one day following the UUO operation (scored as 1; data not shown). Three days later, the damage became evident, as indicated by the presence of enlarged tubules, vacuolar degeneration of tubular epithelial cells, renal interstitial edema and inflammatory cell infiltration (scored as 2; data not shown). Following seven days, these pathological alterations progressed with tubular epithelial necrosis, renal interstitial broadening, and tubular interstitial fibrosis (scored as 3; data not shown). Representative tissue sections with $\mathrm{HE}$ staining are shown in Fig. 1C.

Sham and UUO groups demonstrated significant differences in kidney function. In the majority of cases, the serum and urinary urea nitrogen levels were significantly higher in UUO groups when compared with the Sham group ( $\mathrm{P}=0.027$ and $\mathrm{P}=0.023$ for SUN of UUO1 and UUO3 group vs. Sham group, respectively; $\mathrm{P}=0.018, \mathrm{P}=0.020$ and $\mathrm{P}=0.017$ for $\mathrm{UUN}$ of UUO1, UUO3 and UUO7 group vs. Sham group, respectively; Fig. 2A). Similar results were observed in the serum and urinary creatinine levels $(\mathrm{P}=0.022$ for $\mathrm{SCr}$ of $\mathrm{UUO} 1$ group vs. Sham group; $\mathrm{P}=0.016, \mathrm{P}=0.025$ and $\mathrm{P}=0.019$ for UCr of UUO1, UUO3 and UUO7 group vs. Sham group, respectively; Fig. 2B). These results suggest that kidney dysfunction had been successfully induced in the UUO groups. The significant difference in kidney morphology and kidney function, between Sham and UUO groups suggested 
Table I. Evaluation of KIM-1, $\alpha$-SMA and vimentin expression in Sham, UUO1, UUO3 and UUO7 groups by scoring of immunohistochemical assays.

\begin{tabular}{lccc}
\hline & \multicolumn{3}{c}{ Score } \\
\cline { 2 - 4 } Group & KIM-1 & $\alpha$-SMA & Vimentin \\
\hline Sham & 0 & 0 & 0 \\
UUO1 & 1 & 0 & 0 \\
UUO3 & 2 & 1 & 1 \\
UUO7 & 3 & 2 & 2 \\
\hline
\end{tabular}

KIM-1 was a more sensitive marker of obstructive acute kidney injury when compared with $\alpha$-SMA and vimentin. KIM-1, kidney injury molecule-1; $\alpha$-SMA, $\alpha$-smooth muscle actin; UUO1, 3 and 7, rats at 1, 3 and 7 days following unilateral ureteral obstruction.

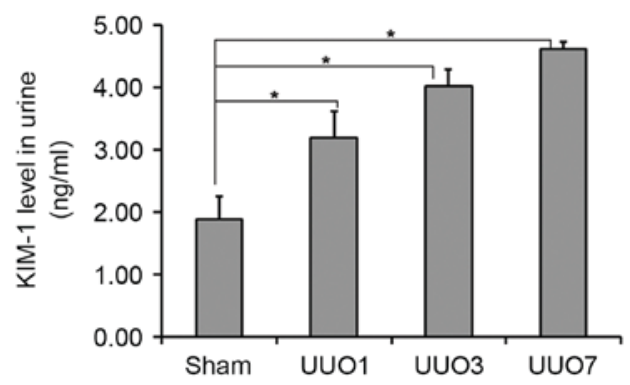

Figure 3. Alterations in KIM-1 levels in the urine. Urinary KIM-1 levels demonstrated a time-dependent increase in rats at 1 (UUO1), 3 (UUO3) and 7 (UUO7) days following UUO. "P<0.05 vs. Sham group. KIM-1, kidney injury molecule-1; UUO, unilateral ureteral obstruction.

that obstructive AKI was successfully induced by the UUO operation.

KIM-1 is a valuable biomarker for the early diagnosis of obstructive AKI. Urinary KIM-1 levels were detected using an ELISA assay. The urinary KIM-1 concentrations of rats in the UUO1, UUO3 and UUO7 groups were $3.20 \pm 0.42$, $4.02 \pm 0.26$ and $4.62 \pm 0.10 \mathrm{ng} / \mathrm{ml}$, respectively; all of which were significantly higher than that of Sham group $(1.89 \pm 0.36 \mathrm{ng} / \mathrm{ml}$; $\mathrm{P}=0.031, \mathrm{P}=0.026$ and $\mathrm{P}=0.015$, respectively; Fig. 3). In addition, urinary KIM-1 levels in the UUO groups displayed a time-dependent increase, correlating with the increasing kidney damage. The same result was subsequently demonstrated with IHC analysis, in which KIM-1 protein expression increased with the increasing area of kidney damage in UUO1, UUO3 and UUO7 groups with IHC scores of 1, 2 and 3, respectively (Table I). Conversely, the Sham group exhibited no obvious KIM-1 expression and was scored as 0 (Fig. 4 and Table I). KIM-1 expression was highly correlated with the development of obstructive AKI, and may therefore be a potential biomarker of obstructive AKI.

To evaluate whether KIM-1 may be a more sensitive marker for early obstructive AKI compared with other biomarkers, the expression of $\alpha$-SMA and vimentin, which have previously been identified as the effective AKI markers (13) were

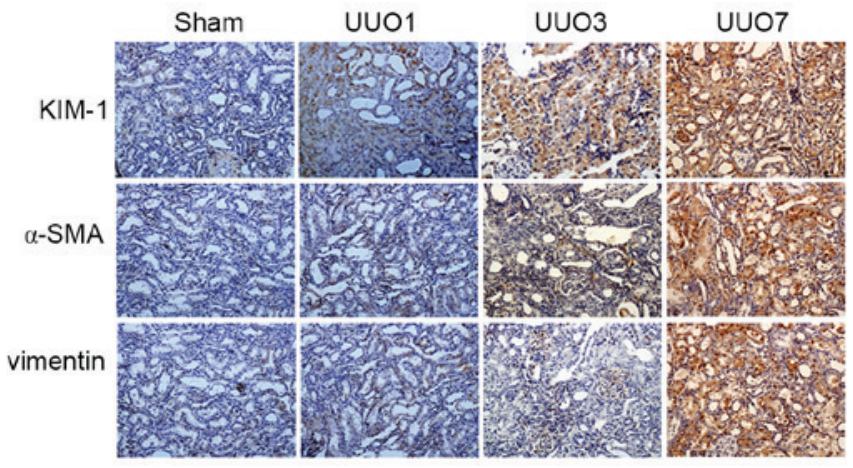

Figure 4. Comparison of KIM-1, $\alpha$-SMA and vimentin expression in kidney tissues of rats at 1 (UUO1), 3 (UUO3) and 7 (UUO7) days following UUO. Representative immunohistochemistry results of KIM-1, $\alpha$-SMA and vimentin protein expression (magnification, $<x 200$ ). Brown staining represents the expression of target proteins. KIM-1, kidney injury molecule-1; $\alpha$-SMA, $\alpha$-smooth muscle actin; UUO, unilateral ureteral obstruction.

examined. The IHC scores are shown in Table I. Similar to $\alpha$-SMA and vimentin, KIM-1 was not detected in the Sham group, but was induced in the UUO groups in a time-dependent manner. Notably, KIM-1 expression was detected in the UUO1 group where obstructive AKI was induced within $24 \mathrm{~h}$ of the UUO operation (Table I). By contrast, $\alpha$-SMA and vimentin were not detected in this group, which indicates that KIM-1 demonstrated a higher sensitivity for the detection of obstructive AKI when compared with the other established biomarkers. Representative results are displayed in Fig. 4. Therefore, KIM-1 was considered to be a valuable biomarker for the early diagnosis of obstructive AKI.

Development of a colloidal gold immunochromatographic strip for the rapid detection of urinary KIM-1. Colloidal gold (pH 7.9) and a McAb of KIM-1 were combined according to the volumes listed in Fig. 5A. Mixtures 2-10 maintained a red color, which meant that these tubes contained enough $\mathrm{McAb}$ KIM-1 to stabilize colloidal gold. Therefore, the minimal volume of McAb KIM-1 (100 $\mu \mathrm{g} / \mathrm{ml})$ required to stabilize $100 \mu 1$ colloidal gold was determined to be between 5 and $10 \mu \mathrm{l}$. In order to identify the optimum volume of KIM-1 required to stabilize $100 \mu \mathrm{l}$ colloidal gold, 6-9 $\mu \mathrm{l}$ of KIM-1 were tested (Fig. 5B). Following 2 h, mixture D maintained its red color, thus the minimal volume of McAb KIM-1 (100 $\mu \mathrm{g} / \mathrm{ml})$ required to stabilize $100 \mu \mathrm{l}$ colloidal gold was determined to be $9 \mu \mathrm{l}$, and the actual volume was calculated as $9 \mu 1 \mathrm{x}(1+10 \%)=9.9 \mu \mathrm{l}$. Based on this volume, a colloidal gold-McAb KIM-1 conjugate solution was obtained and purified for utilization in the preparation of animmunochromatographic strip.

Each component of the immunochromatographic strip was prepared according to the aforementioned methods. The strip was assembled as shown in Fig. 5C. Prior to detection, urine samples were centrifuged at $600 \mathrm{~g}$ for $20 \mathrm{~min}$ at room temperature, and the supernatant was collected for the following test. A detection test was performed by adding the sample to the sample pad of the strip and the results were determined within 5-10 min. Schematic representations of positive, negative and invalid results are shown in Fig. 5D.

To evaluate the sensitivity of the strip, normal human urine was collected and divided into five individual samples, 
A

\begin{tabular}{|c|c|c|c|c|c|c|c|c|c|c|c|}
\hline sample & 0 & 1 & 2 & 3 & 4 & 5 & 6 & 7 & 8 & 9 & 10 \\
\hline Colloidal gold $(\mu \mathrm{l})$ & 100 & 100 & 100 & 100 & 100 & 100 & 100 & 100 & 100 & 100 & 100 \\
\hline McAb KIM-1 ( $\mu \mathrm{l})$ & 0 & 5 & 10 & 15 & 20 & 25 & 30 & 35 & 40 & 45 & 50 \\
\hline $0.005 \mathrm{~mol} / \mathrm{l} \mathrm{NaCl}(\mu \mathrm{l})$ & 50 & 45 & 40 & 35 & 30 & 25 & 20 & 15 & 10 & 5 & 0 \\
\hline $10 \% \mathrm{NaCl}(\mu \mathrm{l})$ & 10 & 10 & 10 & 10 & 10 & 10 & 10 & 10 & 10 & 10 & 10 \\
\hline
\end{tabular}

B

\begin{tabular}{ccccc}
\hline sample & A & B & C & D \\
\hline Colloidal gold $(\mu \mathrm{l})$ & 100 & 100 & 100 & 100 \\
McAb KIM-1 $(\mu \mathrm{l})$ & 6 & 7 & 8 & 9 \\
$0.005 \mathrm{~mol} / \mathrm{I} \mathrm{NaCl}(\mu \mathrm{l})$ & 44 & 43 & 42 & 41 \\
$10 \% \mathrm{NaCl}(\mu \mathrm{l})$ & 10 & 10 & 10 & 10 \\
\hline & & B & &
\end{tabular}

D

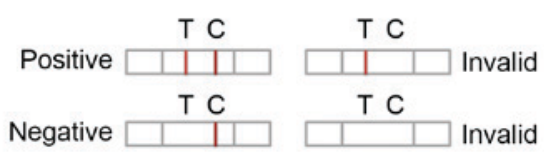

C

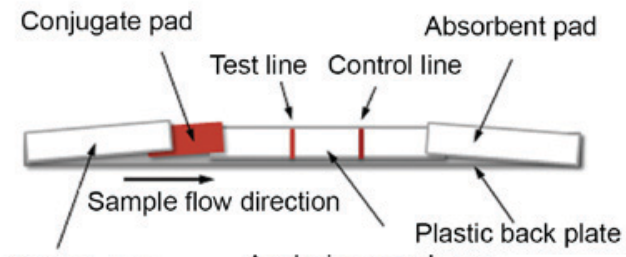

Sample pad Analyzing membrane

$\mathbf{E}$

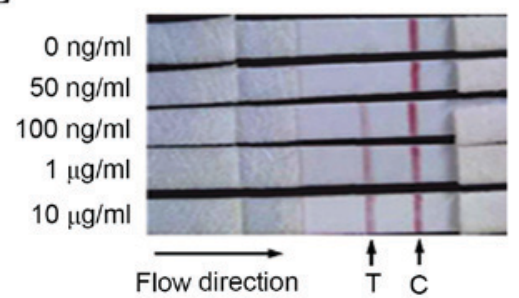

Figure 5. Development of a colloidal gold-based immunochromatographic strip for the rapid detection of urinary KIM-1. Determination of the minimal ratio of McAb KIM-1/colloidal gold with volumes of (A) 0-50 $\mu$ and (B) 6-9 $\mu 1 \mathrm{McAb}$ KIM-1. (C) Components of the colloidal gold immunochromatographic strip and the assembling sequence. (D) Schematic representation of example results. The positive result that Both $\mathrm{T}$ and $\mathrm{C}$ lines are red demonstrates the presence of enough KIM-1 in samples; the negative result that only $\mathrm{C}$ line is in red color suggests the absence or less than the detection limit of KIM-1 in samples; the invalid results, where the $\mathrm{C}$ line is uncolored regardless of the color of $\mathrm{T}$ line, indicates the failure of strips. (E) The detection limit of the strip was determined to be $100 \mathrm{ng} / \mathrm{ml}$. KIM-1, kidney injury molecule-1; McAb, monoclonal antibody; T, goat anti-human KIM-1 polyclonal antibody test line; C, goat anti-mouse IgG control line.

which were spiked with KIM-1 to final concentrations of 0,50 and $100 \mathrm{ng} / \mathrm{ml}$, and 1 and $10 \mu \mathrm{g} / \mathrm{ml}$. The samples were then analyzed using the strip in triplicate, and the representative results are shown in Fig. 5E. Samples with KIM-1 concentrations of $\geq 100 \mathrm{ng} / \mathrm{ml}$ were positive, while samples that contained $<100 \mathrm{ng} / \mathrm{ml}$ KIM-1 were negative. Therefore, the detection limit of the strip was determined to be $100 \mathrm{ng} / \mathrm{ml}$.

\section{Discussion}

Early diagnosis of obstructive AKI remains challenging due to the lack of sensitive and specific biomarkers (14). A recent cohort study indicated that urinary KIM-1 levels were significantly higher in children with severe hydronephrosis when compared with those with mild and non-obstructive forms (9). The authors suggested that increased urinary KIM-1 levels were associated with increased obstruction. An additional study involving 90 patients with obstructive nephropathy demonstrated that urinary KIM-1 content was markedly higher in AKI patients when compared with non-AKI patients (15). In addition, this study indicated that urinary KIM-1 was a long-term predicator of renal outcome in obstructive nephropathy patients with AKI (15). Based on these results, it was hypothesized that urinary KIM-1 may be a useful biomarker for the early diagnosis of obstructive AKI, which is consistent with the results of the present study.

In the current study, UUO rat models were established with sham-operated rats as the control. There was a lower starting body weight in the UUO7 group, which may due to the small sample size, however, rats used in the present study are adult, thus it was assumed that body weight had no decisive effect on parameters included in the current study. Rats in the UUO groups exhibited significant macro- and micro-morphological alterations accompanied by significant kidney dysfunction within hours to days, when compared with the Sham group. This indicated that obstructive AKI was successfully induced in rat models following UUO. The results of the ELISA assays suggested that urinary KIM-1 levels were significantly higher 
in the UUO groups, when compared with the Sham group, and were increased in a time-dependent manner. In addition, IHC assays further confirmed a time-dependent increase in KIM-1 expression in rats from the UUO groups, which was not detected in the kidneys of rats in the Sham group. The results demonstrated a positive correlation between increasing urinary KIM-1 levels and increased obstructive AKI. In addition, KIM-1 was demonstrated to be a more sensitive biomarker of obstructive AKI than $\alpha$-SMA and vimentin, as it was detected during mild obstructive AKI, while $\alpha$-SMA and vimentin was not. The present study provided evidence to suggest that urinary KIM-1 may be a valuable biomarker for the early diagnosis of obstructive AKI.

Although the function of KIM-1 in AKI progression has not yet been completely elucidated (16), assessing KIM-1 levels may provide additional information, when combined with other diagnostic parameters, as early diagnostic biomarkers in preclinical and clinical studies (5). The present study provided evidence for the application of urinary KIM-1 as an early diagnostic biomarker of obstructive AKI, which may be useful for the identification of a therapeutic window during the early stages of the disease. While its applications have not yet reached clinical use, it is necessary to develop an efficient detection method for urinary KIM-1.

Rapidity is a core principle for effective disease diagnosis. The existing detection methods for KIM-1 include IHC, ELISA and western blotting; however these methods do not meet clinical diagnostic requirements due to their time-consuming and complicated detection methods. In the present study, a rapid detection method for KIM-1 was developed, based on the colloidal gold immunochromatographic assay, which detected urinary KIM-1 within 5-10 min. This method has been widely adopted in a number of fields, including medicine, food and pharmaceuticals (17-19). With the advantage of convenience and rapidity, colloidal gold-based immunochromatographic strips are a promising strategy in clinical practice for the rapid diagnosis of AKI. However, in the present study, the detection limit of the strip $(100 \mathrm{ng} / \mathrm{ml})$ was unsatisfactory, thus the sensitivity of the strip requires further optimization. The present study is a preliminary attempt, and future studies will be conducted to improve the sensitivity and specificity of the strip.

In conclusion, the present study confirmed that urinary KIM-1 may be a useful biomarker for the early diagnosis of obstructive nephropathy-induced AKI, which may provide a useful strategy for identifying opportunities for early intervention in the treatment of this disease. In addition, the development of a rapid detection system for urinary KIM-1 was attempted, which provided an insight into the rapid diagnosis of AKI. However, future studies will aim to improve the detection limit via systematic analysis, in order to develop a readily available immunochromatographic strip for the rapid and sensitive detection of urinary KIM-1.

\section{Acknowledgements}

The present study was supported by the Science and Technology Development Project of Jilin Province (grant nos. 20140307006YY and 20150101228JC) and the National Nature Science Foundation of China (grant no. 81302818).

\section{References}

1. Belayev LY and Palevsky PM: The link between acute kidney injury and chronic kidney disease. Curr Opin Nephrol Hypertens 23: 149-154, 2014.

2. Rewa $\mathrm{O}$ and Bagshaw SM: Acute kidney injury-epidemiology, outcomes and economics. Nat Rev Nephrol 10: 193-207, 2014.

3. Susantitaphong P, Cruz DN, Cerda J, Abulfaraj M, Algahtani F, Koulouridis I and Jaber BL; Acute Kidney Injury Advisory Group of the American Society of Nephrology: World incidence of AKI: A meta-analysis. Clin J Am Soc Nephrol 8: 1482-1493, 2013.

4. Moon KH, Ko IK, Yoo JJ and Atala A: Kidney diseases and tissue engineering. Methods 99: 112-119, 2016.

5. Dieterle F, Sistare F, Goodsaid F, Papaluca M, Ozer JS, Webb CP, Baer W, Senagore A, Schipper MJ, Vonderscher J, et al: Renal biomarker qualification submission: A dialog between the FDA-EMEA and predictive safety testing consortium. Nat Biotechnol 28: 455-462, 2010.

6. Ronco C: Acute kidney injury: From clinical to molecular diagnosis. Crit Care 20: 201, 2016.

7. Charlton JR, Portilla D and Okusa MD: A basic science view of acute kidney injury biomarkers. Nephrol Dial Transplant 29: 1301-1311, 2014.

8. Han WK, Bailly V, Abichandani R, Thadhani R and Bonventre JV: Kidney injury molecule-1 (KIM-1): A novel biomarker for human renal proximal tubule injury. Kidney Int 62: 237-244, 2002.

9. Wasilewska A, Taranta-Janusz K, Dębek W, Zoch-Zwierz W and Kuroczycka-Saniutycz E: KIM-1 and NGAL: New markers of obstructive nephropathy. Pediatr Nephrol 26: 579-586. 2011.

10. Wu L, Zhang Y, Ma X, Zhang N and Qin G: The effect of resveratrol on Fox01 expression in kidneys of diabetic nephropathy rats. Mol Biol Rep 39: 9085-9093, 2012.

11. Leelahavanichkul A, Yasuda H, Doi K, Hu X, Zhou H, Yuen PS and Star RA: Methyl-2-acetamidoacrylate, an ethyl pyruvate analog, decreases sepsis-induced acute kidney injury in mice. Am J Physiol Renal Physiol 295: F1825-F1835, 2008.

12. Paek SH, Lee SH, Cho JH and Kim YS: Development of rapid one-step immunochromatographic assay. Methods 22: 53-60, 2000.

13. Ucero AC, Benito-Martin A, Izquierdo MC, Sanchez-Niño MD Sanz AB, Ramos AM, Berzal S, Ruiz-Ortega M, Egido J and Ortiz A: Unilateral ureteral obstruction: Beyond obstruction. Int Urol Nephrol 46: 765-776, 2014.

14. Xie Y, Xue W, Shao X, Che X, Xu W, Ni Z and Mou S: Analysis of a urinary biomarker panel for obstructive nephropathy and clinical outcomes. PLoS One 9: e112865, 2014.

15. Xue W, Xie Y, Wang Q, Xu W, Mou S and Ni Z: Diagnostic performance of urinary kidney injury molecule-1 and neutrophil gelatinase-associated lipocalin for acute kidney injury in an obstructive nephropathy patient. Nephrology (Carlton) 19: 186-194, 2014.

16. Wasung ME, Chawla LS and Madero M: Biomarkers of renal function, which and when? Clin Chim Acta 438: 350-357, 2015.

17. Miyoshi-Akiyama T, Narahara K, Mori S, Kitajima H, Kase T, Morikawa S and Kirikae T: Development of an immunochromatographic assay specifically detecting pandemic H1N1 (2009) influenza virus. J Clin Microbiol 48: 703-708, 2010.

18. Omidfar K, Kia S, Kashanian S, Paknejad M, Besharatie A, Kashanian S and Larijani B: Colloidal nanogold-based immunochromatographic strip test for the detection of digoxin toxicity. Appl Biochem Biotechnol 160: 843-855, 2010.

19. Shim WB, Kim JS, Kim MG and Chung DH: Rapid and sensitive immunochromatographic strip for on-site detection of sulfamethazine in meats and eggs. J Food Sci 78: M1575-M1581, 2013. 